\title{
RFID Tags: Positioning Principles and Localization Techniques
}

\author{
Mathieu Bouet \\ Laboratoire d'Informatique de Paris 6 \\ Université Pierre et Marie Curie \\ Paris, France 75016 \\ mathieu.bouet@lip6.fr
}

\author{
Aldri L. dos Santos \\ Department of Informatics \\ Federal University of Paraná \\ Curitiba, Paraná, Brazil 81531-990 \\ aldri@inf.ufpr.br
}

\begin{abstract}
RFID is an automatic identification technology that enables tracking of people and objects. Both identity and location are generally key information for indoor services. An obvious and interesting method to obtain these two types of data is to localize RFID tags attached to devices or objects or carried by people. However, signals in indoor environments are generally harshly impaired and tags have very limited capabilities which pose many challenges for positioning them. In this work, we propose a classification and survey the current state-of-art of RFID localization by first presenting this technology and positioning principles. Then, we explain and classify RFID localization techniques. Finally, we discuss future trends in this domain.
\end{abstract}

Index Terms-RFID, localization, positioning algorithm.

\section{INTRODUCTION}

Radio Frequency IDentification (RFID) is widely used for electronic identification and tracking. RFID offers substantial advantages for businesses allowing automatic inventory and tracking on the supply chain. This technology plays a key role in pervasive networks and services [1]. Indeed, data can be stored and remotely retrieved on RFID tags enabling realtime identification of devices and users. However, the usage of RFID could be hugely optimized if identification information was linked to location.

This new dimension of context-awareness would support the development of new strategies for autonomic and home networking, mobility control, resource allocation, security, and service discovery algorithms. Such functionalities would also find application in indoor navigation, precise real-time inventory, and in library management to retrieve persons or objects, control access, and monitor events, for example. Classic RFID systems provide coarse-grained location information. Their readers are generally placed in strategic positions, like gates, and their purpose is to detect tags that pass in their read range. Thus, the localization accuracy of such systems corresponds to the dimension of a cell formed by a reader.

With the popularity of indoor location sensing systems and more globally of research on positioning in wireless networks, RFID positioning issue has begun to emerge [2]. Localization methods for RFID tags lie on the same principles than the one for wireless networks. However, they are adapted to the specific capabilities and constraints of this technology. Indeed, tags have very limited capabilities in term of energy and memory providing asymmetric short-range communication and centralized systems. Furthermore, future localization methods will have to consider issues such as reader diversity, mobility, security failures, among others.

This work presents a survey of the state-of-art related to RFID localization. It introduces the current positioning principles for indoor wireless networks in reason of their numerous applications, and a classification of the main RFID localization schemes found in the literature. The article is organized as follows. Section II introduces the RFID technology. Positioning principles for indoor wireless networks are presented in Section III. The RFID localization techniques are presented in Section IV. Finally, Sections V and VI provide perspectives for RFID localization and conclude this survey.

\section{THE RFID TECHNOLOGY}

RFID networks are composed of three different entities, RFID tags, readers, and servers, as shown in Fig. 1. All RFID tags use radio frequency energy to communicate with the readers. However, the method of powering the tags varies. An active tag embeds an internal battery which continuously powers it and its RF communication circuitry. Readers can thus transmit very low-level signals, and the tag can reply with high-level signals. An active tag can also have additional functionalities such as memory, and a sensor, or a cryptography module. On the other hand, a passive tag has no internal power supply. Generally, it backscatters the carrier signal received from a reader. Passive tags have a smaller size and are cheaper than active tags, but have very limited functionalities. The last type of RFID tags is semipassive tags. These tags communicate with the readers like passive tags but they embed an internal battery that constantly powers their internal circuitry.

RFID readers have two interfaces. The first one is a RF interface that communicates with the tags in their read range in order to retrieve tags' identities. The second one is a communication interface, generally IEEE 802.11 or 802.3 , for communicating with the servers.

Finally, one or several servers constitute the third part of an RFID system. They collect tags' identities sent by the reader and perform calculation such as applying a localization 


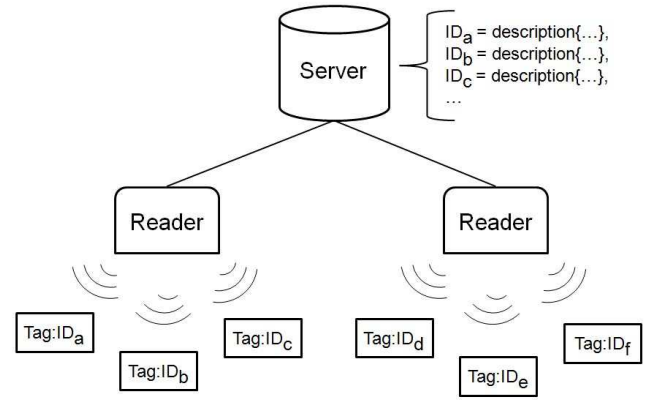

Fig. 1. Architecture of a classic RFID system.

method. They also embed the major part of the middleware system and can be interconnected between each others.

RFID systems can be classified in two main categories in accordance with their usage: monitoring and authorizing [3]. The first class includes RFID systems where tags are attached in an inseparable way to the items they identify. Such networks provide the capability to check, monitor and authenticate which tags are present in the interrogation zone. Classic utilizations are livestock or people embedded with RFID tags. The second class includes RFID systems where RFID tags are not permanently attached to entities. Due to this property, the identity of the entity in possession of the RFID tag cannot be verified. Typical usages of authorizing RFID systems are access control in a building where tags are embedded inside cards or keys.

\section{Positioning PRINCIPLES FOR INDOOR WIRELESS NETWORKS}

Radio propagation in indoor environment is subject to numerous problems such as severe multipath, rare line-ofsight (LOS) path, absorption, diffraction, and reflection [4]. Since signal cannot be measured very precisely, several indoor localization algorithms have been proposed in the literature. They can be classified in three families: distance estimation, scene analysis, and proximity.

\section{A. Distance Estimation}

This family of algorithms uses properties of triangles to estimate the target's location. The triangulation approach, illustrated in Fig. 2, consists in measuring the angle of incidence (or Angle Of Arrival - AOA) of at least two reference points. The estimated position corresponds to the intersection of the lines defined by the angles. On the contrary, the lateration approach, illustrated in Fig. 3, estimates the position of the target by evaluating its distances from at least three reference points. The range measurements techniques use Received Signal Strength (RSS), Time Of Arrival (TOA), Time Difference Of Arrival (TDOA), or Received Signal Phase (RSP).

1) RSS: The attenuation of emitted signal strength is function of the distance between the emitter and the receiver. The target can thus be localized with at least three reference points and the corresponding signal path losses due to propagation. Several empirical and theoretical models have been proposed to translate the difference between the transmitted and the received signal strength into distance estimation. The RSSbased systems usually need on-site adaptation in order to reduce the severe effects of multipath fading and shadowing in indoor environments.

2) TOA: The distance between a reference point and the target is also proportional to the propagation time of signal. TOA-based systems need at least three different measuring units to perform a lateration for 2-D positioning. However, they also require that all transmitters and receivers are precisely synchronized and that the transmitting signals include timestamps in order to accurately evaluate the traveled distances. If more than three reference points are available, the least-squares algorithm or one of its variants can be used in order to minimize the localization error.

3) TDOA: The principle of TDOA lies on the idea of determining the relative location of a targeted transmitter by using the difference in time at which the signal emitted by a target arrives at multiple measuring units. Three fixed receivers give two TDOAs and thus provide an intersection point that is the estimated location of the target. This method requires a precise time reference between the measuring units. Like TOA, TDOA has other drawbacks. In indoor environments, a LOS channel is rarely available. Moreover, radio propagation often suffers from multipath effects thus affecting the time of flight of the signals.

4) RSP: The RSP method, also called Phase Of Arrival (POA), uses the delay, expressed as a fraction of the signal's wavelength, to estimate distance. It requires transmitters placed at particular locations and assumes that they emit pure sinusoidal signals. The localization can be performed using phase measurements and the same algorithm than TOA or phase difference measurements and the same algorithm than TDOA. The disadvantage of the RSP method when applied in indoor environments is that it strongly needs a LOS signal path to limit localization errors.

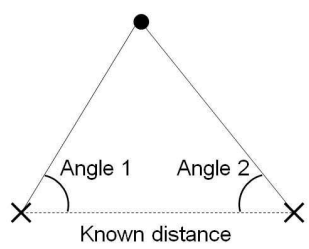

Fig. 2. Triangulation: the estimated location is calculated with the angles formed by two reference points and the target node.

5) $A O A$ : AOA consists in calculating the intersection of several direction lines, each originating from a beacon station or from the target. At least two angles, measured with directional antennae or with an array of antennae and converted in direction lines, are needed to find the 2-D location of a target. Nevertheless, this technique requires complex and expensive equipments and notably suffers from shadowing and multipath reflections.

\section{B. Scene analysis}

Scenes analysis approaches are composed of two distinctive steps. First, information concerning the environment (finger- 


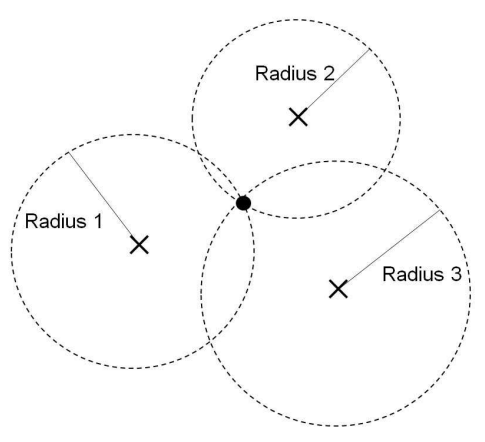

Fig. 3. Trilateration: the estimated location corresponds to the intersection point of three circles.

prints) is collected. Then, the target's location is estimated by matching online measurements with the appropriate set of fingerprints. Generally, RSS-based fingerprinting is used. The two main fingerprinting-based techniques are: k-nearestneighbor $(\mathrm{kNN})$ also known as radio map, and probabilistic methods.

$k N N$ - The $\mathrm{kNN}$ method consists in a first time in measuring RSS at known locations in order to build a database of RSS that is called a radio map. Then, during the online phase, RSS measurements linked to the target are performed to search for the $k$ closest matches in the signal space previously-built. Root mean square errors principle is finally applied on the selected neighbors to find out an estimated location for the target.

Probabilistic Approach - The problem stated in probabilistic approaches is to find the location of a target assuming that there are $n$ possible locations and one observed signal strength vector during the online phase according to posteriori probability and Bayes formula. Thus, the location with the highest probability is chosen. Generally, probabilistic methods involve different stages such as calibration, active learning, error estimation, and tracking with history.

\section{Proximity}

The last type of localization techniques in indoor environments is based on proximity. This approach relies on dense deployment of antennae. When the target enters in the radio range of a single antenna, its location is assumed to be the same that this receiver. When more than one antenna detect the target, the target is assumed to be collocated with the one that receives the strongest signal. This approach is very basic and easy to implement. However, the accuracy is on the order of the size of the cells.

\section{RFID LOCALIZATION SCHEMES}

Several RFID localization methods have been proposed. They utilize the indoor localization principles and are adapted to the characteristics of the RFID technology. Due to the very limited capabilities of tags and contrary to ad-hoc and sensor networks, the localization is always centralized. With passive tags or sparse reader deployment, the proximity approach is privileged. On the contrary, when tags have more energy and thus larger read range or when readers are densely deployed, more elaborated techniques can be applied to localize tags.
RFID localization schemes can be classified into three families: lateration with distance estimation, scene analysis with the deployment of extra reference tags, and constraint-based approach.

\section{A. Distance Estimation}

1) SpotON [5]: SoptON is based on RSS measurements from adjustable long range active RFID tags. The approach is simple: multiple readers collect signal strength measurements in order to approximate distance through a function defined with empirical data. Classic laterations are then performed to localize tags.

2) SAW ID-tags [6]: Surface Acoustic Wave Identification (SAW ID) tags are completely passive. They utilize pulse compression techniques and a large number of coding possibilities. Each tag is interrogated with the time inverse of its impulse response. Then, it retransmits the correlated signal. This retransmitted signal shows an autocorrelation peak. The response with the highest amplitude identifies the searched tag. The distance between each reader $i$ and the tag is measured based on TOA as follows:

$$
d_{i}=\frac{T_{\text {total }, i}-T_{S A W}-T_{\text {sys }}-T_{\text {cable }, i}}{c_{0}} .
$$

The time delay $T_{\text {sys }}$ caused by the system and the time delay $T_{\text {cable }, i}$ due to the cables between each receiving antenna and the demodulator are calculated during a pre-calibration phase. The time delay $T_{S A W}$ is equal for all tags. When three estimated distances are available, the system performs a trilateration to localize the tag.

3) LPM [7]: The Local Position Measurement (LPM) system uses active tags. Since it is based on the TDOA technique, readers are synchronized with the help of reference tags (RT) at well-known and fixed positions that operate continuously. After having received an activation command, the selected measurement tag (MT) responds at time $t_{M T}$. The time difference $t_{d i f f}$ of the corresponding signals at each reader $R_{i}$ can thus be calculated as follows:

$$
c_{0} t_{d i f f}\left(R_{i}\right)=c_{0}\left(t_{M T}-t_{R T}\right)+\left\|M T-R_{i}\right\|-\left\|R T-R_{i}\right\| .
$$

The weighted mean squares method is then utilized to estimate the locations of the tags with at least three different readers.

4) RSP [8]: The authors propose to apply the RSP technique, which they called Direction Of Arrival (DOA), to the localization of passive RFID tags. Their approach consists in placing two readers at specific locations in order to calculate the phase difference and thus the direction of a moving tag. When several observations are available, the estimation can be improved by using the least-square fitting technique. With two pairs of readers, two oblique angles are obtained and utilized for a triangulation calculation as following:

$$
\left(x_{e}, y_{e}\right)=\left(H \cdot \frac{\tan \left(\theta_{1}\right) \tan \left(\theta_{2}\right)}{\tan \left(\theta_{1}\right)+\tan \left(\theta_{2}\right)}, \frac{H}{2} \cdot \frac{\tan \left(\theta_{1}\right)-\tan \left(\theta_{2}\right)}{\tan \left(\theta_{1}\right)+\tan \left(\theta_{2}\right)}\right)
$$

Where $H$ is the distance between the centers of the two arrays formed by the pairs of readers and $\theta_{1}$ and $\theta_{2}$ the estimated DOAs of the tag by the two arrays. 


\section{B. Scene analysis}

1) Landmarc [9]: This system is based on the kNN technique. Reference tags which are fixed tags with known positions are deployed regularly on the covered area. Readers have eight different power levels. This approach consists in selecting the $k$ nearest reference tags from the unknown active tag with the following indicator for each reference tag $j$ :

$$
E_{j}=\sqrt{\sum_{i=1}^{n}\left(\theta_{j, i}-S_{i}\right)^{2}}
$$

Where $n$ is the number of readers, $S_{i}$ the RSS of the tag measured by the reader $i$, and $\theta_{j, i}$ the RSS of the reference tag $j$ measured by the reader $i$. $E$ denotes the relationship between each reference tag and the unknown tag. The $k$ nearest reference tags' coordinates are then used to localize the tag:

$$
\left(x_{e}, y_{e}\right)=\sum_{i=1}^{k} w_{i}\left(x_{i}, y_{i}\right), \text { with } w_{i}=\frac{\frac{1}{E_{i}^{2}}}{\sum_{j=1}^{k} \frac{1}{E_{j}^{2}}} .
$$

The reference tag with the smallest $E$ has the largest weight.

2) VIRE [10]: VIRE uses the principle of Landmarc [9], that is $2 \mathrm{D}$ regular grid of reference tags. Nevertheless, this method introduces the concept of proximity map. The whole sensing area is divided into regions where the center of each region corresponds to a reference tag. Every reader maintains its own proximity map. If the difference between the RSS measurement of the unknown tag and the RSS measurement of a region is smaller than a threshold, the region is marked as ' 1 '. The fusion of all the $n$ readers' maps provides a global proximity map for the tag. Two weighting factors are defined. The first one demonstrates the discrepancy of the RSS measurements between the selected reference tags and the tag:

$$
w_{1 i}=\sum_{j=1}^{n} \frac{\left|\theta_{j, i}-S_{i}\right|}{n \times \theta_{j, i}}
$$

Where $n$ is the number of readers, $S_{i}$ the RSS of the tag measured by the reader $i$, and $\theta_{j, i}$ the RSS of the reference tag $j$ measured by the reader $i$. The second weighting factor is a function related to the density of selected reference tags. The densest area has the largest weight:

$$
w_{2 i}=\frac{p_{i}}{\sum_{j=1}^{n_{a}} p_{j}}
$$

Where $n_{a}$ is the number of total regions and $p_{i}$ denotes the ratio of conjunctive possible regions to the whole area. The coordinates of the tag are finally calculated as following:

$$
\left(x_{e}, y_{e}\right)=\sum_{i=1}^{n_{a}} w_{1 i} \times w_{2 i}\left(x_{i}, y_{i}\right) .
$$

3) Simplex [11]: This method is also based on the deployment of reference tags. It requires that the $n$ readers have $K$ transmission power levels. For the localization of a tag, the readers start with the lowest power level and gradually increase the transmission power until they receive the response from the tag. In the mean time, each reader also receives the responses from reference tags. The distance $L_{i, j}$ between a reader $i$ and a tag $j$ is then estimated by averaging the distances from the reader to all reference tags detected in the same power level but not in the previous power levels. The location of $j$ is calculated by minimizing the error function defined as:

$$
\epsilon_{j}=\sum_{i=1}^{n}\left(\frac{L_{i, j}-\hat{L_{i, j}}}{L_{i, j}}\right)^{2} .
$$

The simplex method is used to minimize $\epsilon_{j}$.

4) Kalman filtering [12]: This approach also utilizes reference tags. The first step consists in calculating with RSS measurements from two readers the distance $D_{i}$ between each reference tag and the target tag. The location of the tag is obtained by solving with the minimum mean squared error algorithm the system of non-linear equations:

$$
\left(x_{i}-x_{e}\right)^{2}+\left(y_{i}-y_{e}\right)^{2}=D_{i}^{2} \quad \forall i=1, \ldots n .
$$

The second step consists in building a probabilistic map of the error measurement for the readers' detection area. The first step is applied for each reference tag in order to calculate their corresponding error probability distribution function with the help of their estimated location and their real location. The Kalman filter is then used iteratively on this online map to reduce the effect of RSS error measurement and thus to improve the accuracy of the localization.

5) Scout [13]: Scout belongs to the family of probabilistic localization techniques. This method also utilizes reference tags and several readers. Active tags are localized following three steps. First, the propagation parameters are calibrated using on-site reference tags. Secondly, the distance between the targeted tag and the readers is estimated with a probabilistic RSS model. Finally, the location of the tag is determined by applying Bayesian inference. Iteratively, predicted beliefs are calculated and then corrected with observations until obtaining a good model resulting in an estimated area.

\section{Constraint-based approach}

1) 3-D Constraints [14]: This approach is only based on connectivity information. They are used to define inclusive constraints, that is if a reader can detect a tag that means that the distance between them is inferior to the read range, and exclusive constraints, the complementary with readers that cannot detect the tag. The space is discretized into points in order to delimit the detection area of the readers. The mean of the set of points that respect the maximum of constraints corresponds to the estimated location of the tag.

Table I briefly compares RFID localization schemes. They are classified according to their approach of the problem. Some of them require the deployment of reference tags which provide finer data but also considerably increase the cost of the system and the maintenance. Except two schemes that are built on very specific properties of passive tags, they all concerns active tags which have larger capacities. Accuracies cannot be directly compared since the systems do not consider the same hypothesis. 
TABLE I

RFID LOCALIZATION SCHEMES

\begin{tabular}{|c||c|c|c|c|}
\hline Localization Scheme & Positioning Algorithm & Reference Tags & Target & Space Dimension \\
\hline SpotON [5] (2000) & RSS lateration & No & Active & 3-D \\
\hline SAW ID-tags [6] (2003) & TOA lateration & No & Passive & 2-D \\
\hline LPM [7] (2004) & TDOA weighted mean squares & No & Active & 2-D \\
\hline RSP [8] (2007) & RSP/AOA & No & Passive & 2-D \\
\hline \hline Landmarc [9] (2003) & kNN & Yes & Active & 2-D \\
\hline VIRE [10] (2007) & kNN & Yes & Active & 2-D \\
\hline Simplex [11] (2007) & kNN optimization & Yes & Active & 3-D \\
\hline Kalman filtering [12] (2007) & RSS mean squares and Kalman filetering & Ycs & 2-D \\
\hline Scout [13] (2006) & RSS Bayesian approach & Yes & Active & 2-D \\
\hline \hline 3-D Constraints [14] (2008) & Range-free optimization & No & Active & 3-D \\
\hline
\end{tabular}

\section{Perspective}

Pervasive networks are potentially rich in term of information. The quantity and the diversity of their components could be used to increase the accuracy of RFID localization. Therefore, future localization methods should take into account several aspects:

- RF model. Most of the current methods perform RSS measurements. However, they generally use models developed for wireless networks. RFID propagation has some particularities that should be considered in an appropriate RF model.

- Reader redundancy. Reader redundancy should be more exploited to obtain more data with the respect of problems in dense deployments of readers [15].

- Reader diversity. Localization in a RFID network with readers that have different read ranges, antennas, and capacities could be an interesting and more realistic approach [16].

- Intelligent constraints. Intelligent constraints could be deduced from meta-information. For example, two tags fixed on the same package define a bound on the physical distance between them [16].

- Mobility. Hybrid systems with static and mobile readers should be considered to increase the quantity and the diversity of collected data.

- Scalability. The scalability of the RFID localization techniques should be carefully studied in order to define the amount of tags that can be read in a given period; the rate of successful reading and its impact on the accuracy; and how long takes the localization calculation.

- Metric. Finally, RFID localization schemes cannot be directly compared since they lie on different hypothesis. An interesting metric to be precisely defined would be the accuracy versus the cost of the whole system.

\section{CONCLUSION}

This paper surveys the current state-of-art of RFID localization. The presented techniques were classified according to their approach: distance estimation, scene analysis, or topological constraints. Designed for passive or active tags, some techniques require the deployment of reference tags when others necessitate specific equipment to take into account changes in environment and to proceed to calibration. On the contrary, some techniques are designed so as to be more cost effective and more easily adaptable to the utilization of different equipments. Globally, in terms of scalability and availability, these RFID positioning techniques have their own important characteristics when applied in real environments. The choice of technique and technology (passive or active tags) significantly affects the granularity and accuracy of the location information but also the whole cost and the efficiency of the RFID system.

\section{REFERENCES}

[1] R. Want. An introduction to RFID technology. IEEE Pervasive Computing, 5(1):25-33, Jan.-March 2006.

[2] A. Cangialosi, J.E. Monaly, and S.C. Yang. Leveraging RFID in hospitals: Patient life cycle and mobility perspectives. IEEE Communications Magazine, 45(9):18-23, Sept. 2007.

[3] T. Hassan and S. Chatterjee. A taxonomy for RFID. In Proc. of HICSS, 2006.

[4] T. Rappaport. Wireless Communications: Principles and Practice. Prentice Hall PTR, Upper Saddle River, NJ, USA, 2001.

[5] J. Hightower, R. Want, and G. Borriello. SpotON: An indoor 3D location sensing technology based on RF signal strength. Technical report, Univ. of Washington, Dep. of Comp. Science and Eng., Seattle, WA, Feb.

[6] T.F. Bechteler and H. Yenigun. 2-D localization and identification based on SAW ID-tags at $2.5 \mathrm{GHz}$. IEEE Trans. on Microwave Theory and Techniques, 51(5):1584-1590, 2003.

[7] A. Stelzer, K. Pourvoyeur, and A. Fischer. Concept and application of LPM - a novel 3-D local position measurement system. IEEE Trans. on Microwave Theory and Techniques, 52(12):2664-2669, Dec. 2004.

[8] Y. Zhang, M. G. Amin, and S. Kaushik. Localization and tracking of passive RFID tags based on direction estimation. International Journal of Antennas and Propagation, 2007.

[9] L.M. Ni, Y. Liu, Y.C. Lau, and A.P. Patil. LANDMARC: indoor location sensing using active RFID. In Proc. of PerCom, pages 407-415, 2003.

[10] Y. Zhao, Y. Liu, and L.M. Ni. VIRE: Active RFID-based localization using virtual reference elimination. In Proc. of ICPP, 2007.

[11] C. Wang, H. Wu, and N.-F. Tzeng. RFID-based 3-D positioning schemes. In Proc. of INFOCOM, pages 1235-1243, 2007.

[12] A. Bekkali, H. Sanson, and M. Matsumoto. RFID indoor positioning based on probabilistic RFID map and kalman filtering. In Proc. of WiMOB, 2007.

[13] X. Huang, R. Janaswamy, and A. Ganz. Scout: Outdoor localization using active RFID technology. In Proc. of BROADNETS, 2006.

[14] M. Bouet and G. Pujolle. A range-free 3-D localization method for RFID tags based on virtual landmarks. In Proc. of PIMRC, 2008.

[15] D.Y. Kim, B.J. Jang, H.G. Yoon, J.S. Park, and J.G. Yook. Effects of reader interference on the RFID interrogation range. In Proc. of European Microwave Conference, pages 728-731, 2007.

[16] N. Vaidya and S.R. Das. Rfid-based networks: exploiting diversity and redundancy. SIGMOBILE Mob. Comput. Commun. Rev., 12(1):2-14, 2008. 